\title{
Derived Cell Line
}

National Cancer Institute

\section{Source}

National Cancer Institute. Derived Cell Line. NCI Thesaurus. Code C156445.

A biospecimen generated by in vitro culturing of a single cell isolated from a tissue of interest. 